\title{
Comparison of two different odorants in an olfactory detection threshold test of the Sniffin' Sticks*
}

\author{
R. Zernecke ${ }^{1}$, B. Vollmer ${ }^{1}$, J. Albrecht ${ }^{1,2}$, A.M. Kleemann ${ }^{1}$, K. Haegler ${ }^{1}$, J. Linn ${ }^{1}$, \\ G. Fesl ${ }^{1}$, H. Brückmann ${ }^{1}$, M. Wiesmann ${ }^{3}$ \\ 1 Department of Neuroradiology, Ludwig-Maximilians-University of Munich, Germany \\ 2 Cognitive Neuroimaging Laboratory, Monell Chemical Senses Center, Philadelphia, PA, USA \\ 3 Department of Neuroradiology, RWTH Aachen, University Aachen, Germany
}

\begin{abstract}
SUMMARY
The olfactory test battery Sniffin' Sticks is a test of nasal chemosensory function that is based on pen-like devices for odour presentation. It consists of three olfactory subtests: threshold, discrimination, and identification. The detection threshold can be measured using two different odorants - n-butanol or PEA (phenylethyl alcohol). Both tasks are commonly applied in published studies, but little is known about the formal comparison of values obtained using them. Unlike the Sniffin' Sticks with n-butanol as odorant, there is poor validation for the threshold subtest with the odorant PEA.

The purpose of this study was to compare these two different odorants. Both odorants were applied to 100 normosmic, healthy subjects (50 females). The experiment was divided into two sessions performed on two different days. After each threshold test the discrimination and identification subtests were conducted.

We obtained significant differences in detection thresholds of PEA and n-butanol. The mean score of PEA threshold and PEA TDI (sum of threshold, discrimination, identification) was significantly higher compared to n-butanol. No significant correlation between individual PEA and n-butanol thresholds was observed.

The differences between both odorants indicate that a formal validation of the Sniffin' Sticks with PEA as odorant for probing olfactory thresholds may be required.
\end{abstract}

Key words: n-butanol, phenylethyl alcohol, sensitivity, chemosensory perception

\section{INTRODUCTION}

Olfactory tests are very important for clinical diagnosis of otorhinolaryngologic and neurological disorders as well as for experimental research ${ }^{(1-4)}$. Especially, odour detection threshold tests are used for clinical evaluation of patients with neurological disorders ${ }^{(1,5,6)}$, and to assess olfactory function in psychophysical studies $^{(7-9)}$.

Tests for the assessment of olfactory function have been numerously described in the literature ${ }^{(10)}$. In clinical practice, few of them are actually used because of inconsistency of some tests, poor validation, time needed for administration, and their limited availability ${ }^{(11)}$. The most widely known instruments currently available for olfactory testing are the "University of Pennsylvania Smell Identification Test" (UPSIT) ${ }^{(12,13)}$, its down-scaled version the "Cross Cultural Smell Identification Test" (CC-SIT) ${ }^{(14)}$, the "Connecticut Chemosensory Clinical Research Center Test" (CCCRC) ${ }^{(15,16)}$, and the olfactory test battery "Sniffin' Sticks" ${ }^{(17,18)}$. Most of these olfactory tests are positively correlated with each other and measure common attributes ${ }^{(19)}$.

The Sniffin' Sticks test is a well-established test battery for combined testing of olfactory function. It comprises three subtests, namely odour threshold, odour discrimination, and odour identification using pen-like odour-dispensing devices for odour presentation. It was introduced by Kobal et al. ${ }^{(17)}$ and Hummel et al. ${ }^{(18)}$ in Germany. The Sniffin' Sticks test battery is in daily use by many practitioners all across Europe ${ }^{(20)}$, but it is also used in Australia and North America for olfactory testing ${ }^{(9,21)}$. For the Australian population, normative data has been published by Mackay-Sim et al. ${ }^{(21)}$. In Korea, a modified version of the Sniffin' Sticks is commercially available (Korean version of the Sniffin' Stick, KVSS I and KVSS II) which was recently validated in comparison with the CC-SIT ${ }^{(22)}$.

Most of the studies mentioned above were carried out with the Sniffin' Sticks using n-butanol as odour detection threshold task. This test battery has been thoroughly validated; norma- 
tive data are based on investigations in more than 3000 subjects ${ }^{(23,24)}$. Besides the $n$-butanol detection threshold test, another detection threshold test of the Sniffin' Sticks is in common use. The threshold can also be measured using phenylethyl alcohol (PEA) as odorant. In comparison to nbutanol, PEA has a pleasant rose-like smell and produces little, if any, trigeminal sensations ${ }^{(25)}$. Therefore, PEA is frequently selected as a test odorant ${ }^{(9,26-30)}$ but little is known about the validity of the PEA threshold test ${ }^{(29)}$, in contrast to the Sniffin' Sticks with n-butanol as odorant for detecting the olfactory threshold. Croy et al. ${ }^{(31)}$ compared these two odour threshold subtests in 116 subjects using different numbers of dilution steps. They did not observe significant differences between the n-butanol and the PEA threshold tests, and found a significant correlation between both odorants. In spite of the findings of Croy et al. ${ }^{(31)}$, in the present study we conducted a formal comparison of the two different odorants, PEA and n-butanol, in an olfactory detection threshold subtest of the Sniffin' Sticks, using the common staircase method.

\section{MATERIAL AND METHODS}

Subjects

A total of 104 normosmic, healthy subjects attended in this study. Four participants were excluded because they had a cold in between the interval of the two sessions. Therefore, 100 subjects were included for statistical analysis (50 males, 50 females; age range 18-51 years: mean age 26.42 years, SD 6.40 years; age range 18-35 years: mean 24.64 years, SD 3.81 years, $\mathrm{n}=89$; age range $36-51$ years: mean 40.82 years, SD 4.56 years, $\mathrm{n}=11)$.

Using the screening test Sniffin' Sticks with thoroughly validated n-butanol as odour detection threshold, all subjects were tested for normal olfactory function ${ }^{(18,23,24)}$. Mean age did not differ significantly between male (mean age 27.14 years, SD 7.52 years) and female (mean age 25.70 years, SD 5.01 years) subjects (Mann-Whitney-U-Test: $\mathrm{p}=$ n.s.). All subjects were non-smokers and were not taking any medication known to interfere with sensory perception ${ }^{(32-34)}$. None of them reported any olfactory disturbances. None of the female subjects reported to be pregnant or to lactate.

The study protocol was approved by the Medical Ethics Review Committee of the Ludwig-Maximilians-University of Munich, Germany. This study was performed at the Department of Neuroradiology of this University. All participants provided a written informed consent.

\section{Stimulus material}

Olfactory testing was performed by means of the Sniffin' Sticks test battery (Burghart Instruments, Wedel, Germany) ${ }^{(17,18)}$ which comprises three subtests, namely odour threshold, odour discrimination, and odour identification. In the present study we compared two different olfactory detection threshold tests available for the Sniffin' Sticks. The threshold part of the Sniffin' Sticks assesses subject's sensitivity to either the odor- ant n-butanol or phenylethyl alcohol (PEA). Detection thresholds were determined using a single-'staircase', three-alternative forced-choice procedure described by Doty ${ }^{(35)}$.

\section{Experimental procedures}

For threshold measurements, odorants (n-butanol and PEA) were assessed in sixteen different dilutions starting with the lowest concentration. Both odorants were diluted in geometric series consisting of sixteen steps with a dilution ratio of 1:2; the highest administered concentration of n-butanol and PEA was $4 \% \mathrm{v} / \mathrm{v}$, respectively. The odour n-butanol was diluted in aqua conservata (demineralized water with antidegradants) and PEA was diluted in propylene glycol. Three sticks were presented in a randomized order; two of them contained only the solvent (aqua conservata or propylene glycol) serving as blanks and the third contained the odour (n-butanol or PEA) at a certain dilution. Participants had to identify the odour-containing pen. Two successful identifications in a row or one unsuccessful identification triggered a reversal of the staircase to the next higher or next lower dilution, respectively. Threshold score was defined as the mean of the last four out of seven staircase reversals and ranged from 1 (lowest sensitivity, highest threshold) to 16 (highest sensitivity, lowest threshold).

In the odour discrimination task, sixteen triplets of odorants were presented in randomized order. In each triplet two sticks contained the same odour and the third contained a different odour. Participants were required to determine which of the three odour-containing sticks smelled differently compared to the other two sticks. Resulting scores ranged from 0 (no correct discrimination) to 16 (perfect discrimination).

Odour identification was assessed by means of sixteen commonly known every day odorants in randomized order. Using a multiple choice task, identification of individual odours was performed from lists of four descriptors each. Resulting scores ranged from 0 (no correct identification) to 16 (perfect identification).

From the results of the odour threshold, odour identification, and odour discrimination tasks, a composite TDI score (sum of threshold, discrimination, and identification scores) was derived. The TDI score was used to determine normal olfactory function. The subjects' TDI scores could range from 1 to 48 . Normosmia was defined as a TDI score of $>30^{(18,23,24,36)}$.

All subtests were assessed birhinally. During the threshold and discrimination tasks subjects were blindfolded to prevent any visual cues during testing.

Using the Sniffin' Sticks test battery, olfactory sensitivity (detection threshold) was tested first followed by odour discrimination and odour identification to assess subject's olfactory function. Standard procedure for using this test battery is described by Kobal et al. ${ }^{(17)}$ and Hummel et al. ${ }^{(18)}$. All tests 
were conducted with the Sniffin' Sticks testing software named Olaf $^{(37)}$.

Both odorants (n-butanol and PEA) of the olfactory detection threshold subtest were presented in two sessions performed on two different days. For each participant both sessions were carried out at the same time of the day. The interval between both sessions was between one and ten days (mean 4.11 days, SD 2.72 days). Order of threshold testing (n-butanol or PEA) was pseudo randomized and ratio of gender was systematically counterbalanced.

Since odour discrimination and odour identification tests were performed twice during the experiment (session 1 and 2), the sticks of each subtest were administered in randomized order to prevent learning effects by the participants.

After each session subjects completed a questionnaire. One session of the experiment lasted approximately half an hour.

\section{Questionnaire}

A questionnaire consisting of eight parameters was employed to measure the perception of the Sniffin' Sticks, the recipients' emotional state, and their current state of hunger. After each session, subjects rated their current state of hunger $(0=$ very hungry, $100=$ not hungry at all; $0=$ no appetite, $100=$ strong appetite; $0=$ empty stomach, $100=$ filled stomach), their emotional valence $(0=$ positive, $100=$ negative $)$, their arousal $(0=$ aroused, $100=$ calm), and their alertness $(0=$ very inattentive, $100=$ very attentive) by using a visual analog scale (VAS). Furthermore, subjects rated the pleasantness $(0=$ pleasant, 100 = unpleasant) of the odorants n-butanol and PEA, and the intensity ( $0=$ very weak, $100=$ very strong) of the pen containing the highest concentration of $n$-butanol (4\% v/v) and PEA $(4 \% \mathrm{v} / \mathrm{v})$.

Table 1. Mean scores and standard deviations of the olfactory subtests and the composite TDI score of the Sniffin' Sticks, and means and standard deviations of the eight parameters of the questionnaire of both test sessions (interval $\leq 10$ days). Asymptotic significance (2tailed) of Wilcoxon signed-rank test of the comparison of two odorants (n-butanol versus PEA) regarding these parameters $(n=100)$.

\begin{tabular}{lccc}
\hline & $\begin{array}{c}\text { n-butanol } \\
\text { session }\end{array}$ & $\begin{array}{c}\text { PEA } \\
\text { session }\end{array}$ & $\begin{array}{c}\text { Wilcoxon } \\
\text { signed-rank test }\end{array}$ \\
\hline Threshold & $8.91(1.71)$ & $11.64(2.50)$ & $0.000^{*}$ \\
Discrimination & $13.50(1.49)$ & $13.42(1.62)$ & 0.972 \\
Identification & $13.69(1.23)$ & $13.52(1.49)$ & 0.161 \\
TDI & $36.11(2.53)$ & $38.59(3.29)$ & $0.000^{*}$ \\
Valence & $29.09(18.86)$ & $28.93(17.90)$ & 0.814 \\
Arousal & $77.10(18.52)$ & $77.32(18.73)$ & 0.706 \\
Alertness & $78.61(13.73)$ & $78.50(12.75)$ & 0.796 \\
Intensity & $77.06(18.27)$ & $73.37(20.32)$ & 0.189 \\
Pleasantness & $54.43(21.01)$ & $22.53(15.11)$ & $0.000^{*}$ \\
Hungriness & $60.01(22.76)$ & $59.32(22.47)$ & 0.532 \\
Appetite & $32.08(23.83)$ & $31.26(24.48)$ & 0.953 \\
Fullness of stomach & $49.28(21.49)$ & $48.97(22.16)$ & 0.561 \\
\hline
\end{tabular}

* Wilcoxon signed-rank test significant with $\mathrm{p}<0.001$.

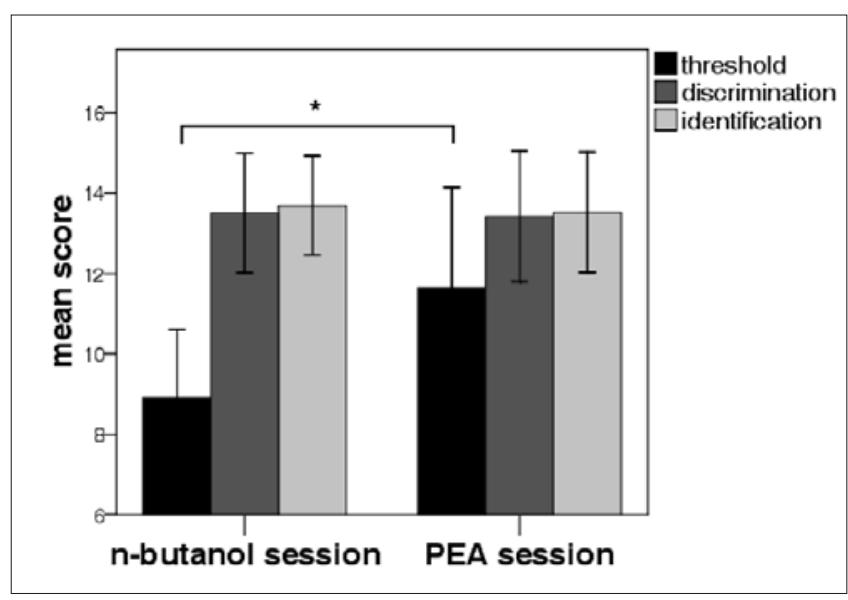

Figure 1. Mean scores and standard deviations of the olfactory subtests of the Sniffin' Sticks of two test sessions using n-butanol and PEA as odorants in the detection threshold task $(\mathrm{n}=100)$. * Wilcoxon signedrank test significant with $\mathrm{p}<0.001$.

The questions were answered by the participants using a VAS. They gave a response by placing a mark on a $100 \mathrm{~mm}$ horizontal line. VAS have been shown to measure even minor changes in affect with high reliability and validity ${ }^{(38,39)}$.

\section{Statistical analyses}

Data were statistically analyzed using SPSS version 16.0 for Windows (SPSS Inc., Chicago, IL, USA). Normality of the data was tested using the Kolmogorov-Smirnov test. The non-parametric Wilcoxon signed-rank test was used to explore differences between the odorants, n-butanol and PEA. Results were illustrated in histograms with means and standard deviations. Correlation analyses were performed using Spearman's rank test to examine the relationship between both odorants. This correlation was represented in a scatter plot. To analyze gender differences data were submitted to a Mann-Whitney U test. Results of the questionnaire were corrected for multiple testing using the Bonferroni method. The alpha level was set at 0.05 .

\section{RESULTS}

Olfactory sensitivity and TDI score

The results of the two different detection threshold tasks of the Sniffin' Sticks differed significantly $(\mathrm{p}<0.001)$. Mean olfactory detection threshold was 8.91 (SD 1.71) for n-butanol, and 11.64 (SD 2.50) for PEA (Table 1, Figure 1). Participants detected PEA at a lower concentration than n-butanol. Comparison of TDI scores obtained using the two different sets of the Sniffin' Sticks (n-butanol versus PEA as odour detection threshold) also revealed significant differences $(\mathrm{p}<$ 0.001). Mean TDI score of the olfactory test battery using PEA as odour detection threshold was significantly higher (38.59, SD 3.29; range 31.50-46.50) compared to the test battery using n-butanol (36.11, SD 2.53; range 31.00-43.75) (Table 1).

Correlation analyses of both olfactory detection threshold tasks revealed no significant correlation coefficient (Spearman-rho 


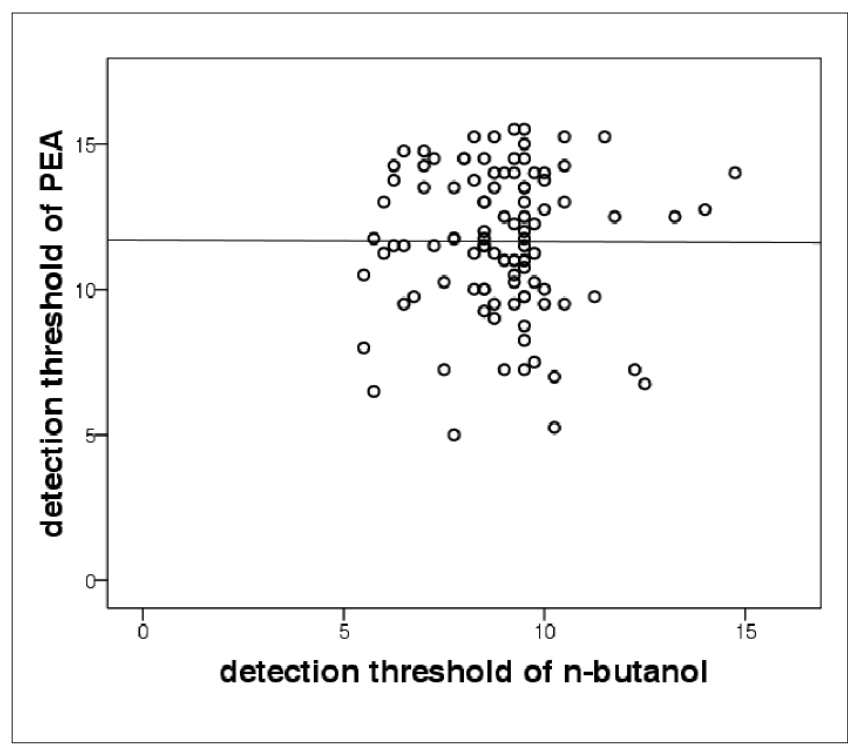

Figure 2. Correlation between the results of the olfactory detection threshold test utilizing two different odorants, $\mathrm{n}$-butanol and PEA ( $\mathrm{n}=$ 100). Spearman-rho correlation coefficient $=-0.039, \mathrm{p}=0.699$ (n.s.).

correlation coefficient $=-0.039 ; \mathrm{p}=\mathrm{n} . \mathrm{s}$.) (Figure 2). No significant correlation between individual PEA and n-butanol sensitivity was observed.

With regard to the variable gender, there were significant differences in olfactory detection thresholds of the odour nbutanol ( $\mathrm{p}=0.037$ ), with females (mean 9.24, SD 1.86) being more sensitive to n-butanol than males (mean 8.59, SD 1.48). The odorant PEA was also detected at a lower concentration by women (mean 11.87, SD 2.65) when compared to men (mean 11.41, SD 2.34) but the difference was not significant ( $p$ $=$ n.s.). The TDI score did not differ significantly between male and female subjects $(\mathrm{p}=$ n.s.).

\section{Olfactory discrimination and olfactory identification}

No significant differences in olfactory discrimination $(p=n . s$.) as well as olfactory identification tasks $(p=n$.s.) between the two sessions were observed (Table 1, Figure 1). The subtests yielded similar results in both test sessions starting with two different olfactory detection threshold tasks (n-butanol and PEA). There were no gender-related differences for these olfactory subtests $(\mathrm{p}=$ n.s.). Descriptive statistics are shown in Table 1.

\section{Rating of odorants, emotional conditions, and current state of} hunger

Mean ratings of both odorants differed significantly with respect to pleasantness $(\mathrm{p}<0.001)$. Participants evaluated the odorant PEA as more pleasant (mean 22.53, SD 15.11) compared to n-butanol (mean 54.43, SD 21.01) (Table 1). Mean ratings of the other parameters did not differ between both sessions. There were no significant differences in emotional conditions (valence, arousal, alertness) and subjects' evaluations of their current state of hunger (hungriness, appetite, fullness of stomach) between both testing days ( $p=$ n.s.). The intensity of the pen containing the highest concentration of n-butanol and PEA were also rated similar in both measurements $(\mathrm{p}=\mathrm{n} . \mathrm{s}$. $)$. Descriptive statistics are shown in Table 1.

\section{DISCUSSION}

In the present study, the comparison of two different odorants utilized in the threshold test of the Sniffin' Sticks revealed different results in subjects' olfactory sensitivities and TDI scores, whereas discrimination and identification tests yielded similar results across both testing days.

Unlike the findings of Croy et al. ${ }^{(31)}$, our results of detection thresholds of n-butanol with a mean score of $8.91^{(18,23,24)}$ and of PEA with a mean score of $11.64^{(9,29,30)}$ were similar compared to previous results. The mean score of PEA (11.87) reported by Croy et al. ${ }^{(31)}$ was similar to our and previous findings of this detection threshold test, whereas their results of a mean score of n-butanol (11.52) are not in line with the existing literature. The mean TDI score using PEA for detection threshold in the present study was also significantly higher compared to nbutanol because the TDI score is a composite score of results obtained in each subtest of the Sniffin' Sticks ${ }^{(23,36)}$.

At first sight, it may appear surprising that we did not find a significant correlation between the subjects' individual sensitivities to PEA and n-butanol. Most importantly, this raises the question if the Sniffin' Sticks test battery is a poor test for assessing subjects' olfactory function, meaning it possesses a low test-retest reliability. This, however, is not true. Several studies, including one of our own group, have shown that the Sniffin' Sticks with n-butanol as detection threshold task retains a high test-retest reliability ${ }^{(17,18,40)}$. Why then did we not find a correlation between the two odorants whereas Croy et al. ${ }^{(31)}$ observed a significant correlation? Comparing olfactory sensitivities between different odorants will in most cases yield at least some correlation within subjects. This is all the more true when subjects of a higher age range (19-74 years) or subjects who present with olfactory impairment are included as Croy et al. ${ }^{(31)}$ did. However, Croy et al. ${ }^{(31)}$ presented no results for correlations regarding normosmic subjects exclusively. Hyposmic patients will show a lower olfactory sensitivity than normosmic subjects regardless of the test used, and thus it is not surprising that comparisons between olfactory tests usually show high correlations when sensitivity of both healthy and impaired subjects is investigated. On the other hand, the more homogeneous a group of subjects is with regard to their olfactory performance, the more important other factors do become. A subject's individual sensitivity to a certain odour depends mainly on the subject's receptor repertoire. Naturally, all humans differ in their olfactory receptor repertoire. If the subsets required for detecting two different odours do widely overlap, the performance of normosmic subjects will be highly correlated. This will often be the case, and thus, it is not surprising that there are studies showing that in normal subjects 
the detection thresholds between different odours are correlated ${ }^{(41)}$. However, for any two given odorants, this is possible but it does not need to be the case. There are even odorants for which a considerable proportion of normosmic participants are selectively anosmic. Since the two odorants PEA and nbutanol have not been compared in normosmic subjects before, and since we have used a methodology which in a previous study has yielded a high test-retest reliability of the Sniffin' Sticks ${ }^{(40)}$, our results suggest, that n-butanol and PEA are detected by subsets of olfactory receptors, which show a comparably small degree of receptor overlap. Therefore, subjects showed different sensitivities to both odorants.

Different odorants, like n-butanol and PEA, are recognized by different combinations of olfactory receptors ${ }^{(42-45)}$. Humans may have a better receptor repertoire for PEA than for n-butanol. As Keller et al. ${ }^{(46)}$ showed that the perception of odorants is related to human olfactory receptors and their variations in genotypes. Thus, olfactory receptors affect not only the detection of odorants, but also the evaluation of pleasantness and intensity. This link between subjective perception and detection of odorants is, furthermore, supported by the neuronal connection to the limbic system when olfactory stimuli are processed ${ }^{(47,48)}$. Emotional evaluation of an odorant may influence subject's motivation and in consequence odour detection threshold. Gudziol et al. ${ }^{(49)}$ demonstrated that participants inhaled pleasant odours more deeply and for a longer duration. In our study, subjects' inhalation of PEA could have been longer and deeper than participants' inhalation of n-butanol, which in consequence could have influenced their olfactory sensitivities.

The solvents that are used to dilute the odorants could also have influenced subjects' olfactory sensitivities. Differences in detection thresholds of PEA have been reported in literature when different solvents were used ${ }^{(50,51)}$. In our study, the two different solvents could have activated different olfactory receptors, additionally, and thus influenced subjects' sensitivity to n-butanol and PEA. Furthermore, different solvents affect the vapour concentrations, which in consequence make it difficult to compare these two odorants directly. Therefore, the lack of correlation is not surprising. A profound validation of the subtest with PEA as odorant is indicated.

Our study revealed differences between the threshold tests of the Sniffin' Sticks using two different threshold odorants, nbutanol and phenylethyl alcohol. Our data confirm previous reports that the Sniffin' Sticks with n-butanol as detection threshold task is an undisputable well established test. Our results are in line with previously published mean scores of both detection threshold subtests unlike the findings of Croy et al. ${ }^{(31)}$ that are not in accordance with the literature. The observed differences in both threshold subtests could reflect variances in olfactory function. Different odours influence the nasal mucosa and the perception of odours in different ways. In the present study, this could have influenced the olfactory sensitivity of subjects.

Both odours used for olfactory detection threshold testing performed equally well in their assessments. However, the scores achieved in the detection threshold test as well as the composite TDI scores differed, depending on whether n-butanol or PEA was selected as odorant. This has implications on the clinical and scientific use of the Sniffin' Sticks with PEA as odorant. It may be appropriate to modify the TDI scale for assessing normosmia if PEA instead of n-butanol is used for the threshold test. Both olfactory test batteries are a comfortable and simple method for clinical as well as research testing of olfactory performance. The Sniffin' Sticks with n-butanol as detection threshold task is already validated. A profound confirmation of the Sniffin' Sticks with PEA as odorant has not been probed, yet. An expanded database including healthy participants of a widespread age range, as well as patients, but also studies about the test-retest reliability, and subsequent modifications of the Sniffin' Sticks threshold subtest with the odorant PEA is required and should be investigated in further studies.

\section{ACKNOWLEDGMENTS}

Parts of this study were developed in line with the dissertation of Birgit Vollmer at the Medical Faculty of the LudwigMaximilians-University of Munich (in preparation).

\section{REFERENCES}

1. Daum RF, Sekinger B, Kobal G, et al. Olfactory testing with "sniffin' sticks" for clinical diagnosis of Parkinson disease. Nervenarzt 2000; 71: 643-650.

2. Hummel T, Konnerth CG, Rosenheim K, et al. Screening of olfactory function with a four-minute odor identification test: reliability, normative data, and investigations in patients with olfactory loss. Ann Otol Rhinol Laryngol 2001; 110: 976-981.

3. Wrobel BB, Leopold DA. Clinical assessment of patients with smell and taste disorders. Otolaryngol Clin North Am 2004; 37 : 1127-1142.

4. Elsherif HS, Landis BN, Hamad MH, et al. Olfactory function and nasal nitric oxide. Clin Otolaryngol 2007; 32: 356-360.

5. Doty RL, Reyes PF, Gregor T. Presence of both odor identification and detection deficits in Alzheimer's disease. Brain Res Bull 1987; 18: 597-600.

6. Murphy C, Gilmore MM, Seery CS, et al. Olfactory thresholds are associated with degree of dementia in Alzheimer's disease. Neurobiol Aging 1990; 11: 465-469.

7. Doty R, Gregor T, Settle RG. Influence of intertrial interval and sniff-bottle volume on phenyl ethyl alcohol odor detection thresholds. Chem Senses 1986; 11: 259-264.

8. Heywood PG, Costanzo RM. Identifying normosmics: a comparison of two populations. Am J Otolaryngol 1986; 7: 194-199.

9. Lundstrom JN, Boyle JA, Jones-Gotman M. Sit up and smell the roses better: olfactory sensitivity to phenyl ethyl alcohol is dependent on body position. Chem Senses 2006; 31: 249-252.

10. Doty RL. Office procedures for quantitative assessment of olfactory function. Am J Rhinol 2007; 21: 460-473.

11. Doty RL, Laing DG. Psychophysical measurements of human olfactory function, including odorant mixture assessment. In: Doty RL, editor. Handbook of olfaction and gustation. 2nd ed. New York: Marcel Dekker; 2003. p. 203-28. 
12. Doty RL, Shaman P, Dann M. Development of the University of Pennsylvania Smell Identification Test: a standardized microencapsulated test of olfactory function. Physiol Behav 1984; 32: 489502.

13. Doty RL, Shaman P, Kimmelman CP, et al. University of Pennsylvania Smell Identification Test: a rapid quantitative olfactory function test for the clinic. Laryngoscope 1984; 94 (2 Pt 1): 176-178.

14. Doty RL, Marcus A, Lee WW. Development of the 12-item CrossCultural Smell Identification Test (CC-SIT). Laryngoscope 1996; 106 (3 Pt 1): 353-356.

15. Cain WS, Gent JF, Goodspeed RB, et al. Evaluation of olfactory dysfunction in the Connecticut Chemosensory Clinical Research Center. Laryngoscope 1988; 98: 83-88.

16. Cain WS. Testing olfaction in a clinical setting. Ear Nose Throat $\mathrm{J}$ 1989; 68: 316; 322-328.

17. Kobal G, Hummel T, Sekinger B, et al. "Sniffin' sticks": screening of olfactory performance. Rhinology 1996; 34: 222-226.

18. Hummel T, Sekinger B, Wolf SR, et al. 'Sniffin' sticks': olfactory performance assessed by the combined testing of odor identification, odor discrimination and olfactory threshold. Chem Senses 1997; 22: 39-52.

19. Frank RA, Dulay MF, Gesteland RC. Assessment of the Sniff Magnitude Test as a clinical test of olfactory function. Physiol Behav 2003; 78: 195-204

20. Huttenbrink KB. Disorders of smell and taste. Standard and recent methods in diagnosis and therapy. Laryngorhinootologie 1997; 76 : 506-514.

21. Mackay-Sim A, Grant L, Owen C, et al. Australian norms for a quantitative olfactory function test. J Clin Neurosci 2004; 11: 874879.

22. Cho JH, Jeong YS, Lee YJ, Hong SC, Yoon JH, Kim JK. The Korean version of the Sniffin' stick (KVSS) test and its validity in comparison with the cross-cultural smell identification test (CCSIT). Auris Nasus Larynx 2008; 36: 280-286.

23. Kobal G, Klimek L, Wolfensberger M, et al. Multicenter investigation of 1,036 subjects using a standardized method for the assessment of olfactory function combining tests of odor identification, odor discrimination, and olfactory thresholds. Eur Arch Otorhinolaryngol 2000; 257: 205-211.

24. Hummel T, Kobal G, Gudziol H, et al. Normative data for the "Sniffin' Sticks" including tests of odor identification, odor discrimination, and olfactory thresholds: an upgrade based on a group of more than 3,000 subjects. Eur Arch Otorhinolaryngol 2007; 264: 237-243.

25. Doty RL, Brugger WE, Jurs PC, et al. Intranasal trigeminal stimulation from odorous volatiles: psychometric responses from anosmic and normal humans. Physiol Behav 1978; 20: 175-185.

26. Kobal G, Palisch K, Wolf SR, et al. A threshold-like measure for the assessment of olfactory sensitivity: the "random" procedure. Eur Arch Otorhinolaryngol 2001; 258: 168-172.

27. Knecht M, Lundstrom JN, Witt M, et al. Assessment of olfactory function and androstenone odor thresholds in humans with or without functional occlusion of the vomeronasal duct. Behav Neurosci 2003; 117: 1135-1141.

28. Lundstrom JN, Hummel T, Olsson MJ. Individual differences in sensitivity to the odor of 4,16-androstadien-3-one. Chem Senses 2003; 28: 643-650.

29. Lotsch J, Lange C, Hummel T. A simple and reliable method for clinical assessment of odor thresholds. Chem Senses 2004; 29: 311317.

30. Pollatos O, Albrecht J, Kopietz R, et al. Reduced olfactory sensitivity in subjects with depressive symptoms. J Affect Disord 2007; 102 (1-3): 101-108.

31. Croy I, Lange K, Krone F, et al. Comparison between odor thresholds for phenyl ethyl alcohol and butanol. Chem Senses 2009; 34(6): 523-527.
32. Frye RE, Schwartz BS, Doty RL. Dose-related effects of cigarette smoking on olfactory function. Jama 1990; 263: 1233-1236.

33. Schiffman S. Changes in taste and smell: drug interactions and food preferences. Nutr Rev 1994; 52 (8 Pt 2): 11-14.

34. Doty RL, Bromley SM. Effects of drugs on olfaction and taste. Otolaryngol Clin North Am 2004; 37: 1229-1254

35. Doty RL. Olfactory system. In: Getchell TV, Doty RL, Bartoshuk LM, Snow JB, Jr, editors. Smell and taste in health and disease. New York: Raven Press; 1991. p. 175-199.

36. Wolfensberger M, Schnieper I, Welge-Lussen A. Sniffin'Sticks: a new olfactory test battery. Acta Otolaryngol 2000; 120: 303-306.

37. Hummel T. Olfactory testing using the Sniffin Sticks. http://www.tu-dresden.de/medkhno/riechen_schmecken/download.htm. 2009.

38. Aitken RC. Measurement of feelings using visual analogue scales. Proc R Soc Med 1969; 62: 989-993.

39. Folstein MF, Luria R. Reliability, validity, and clinical application of the Visual Analogue Mood Scale. Psychol Med 1973; 3: 479-486.

40. Albrecht J, Anzinger A, Kopietz R, et al. Test-retest reliability of the olfactory detection threshold test of the Sniffin' sticks. Chem Senses 2008; 33: 461-467.

41. Cain WS, Gent JF. Olfactory sensitivity: reliability, generality, and association with aging. J Exp Psychol Hum Percept Perform 1991; 17: 382-391.

42. Malnic B, Hirono J, Sato T, et al. Combinatorial receptor codes for odors. Cell 1999; 96: 713-723.

43. Touhara K. Odor discrimination by $\mathrm{G}$ protein-coupled olfactory receptors. Microsc Res Tech 2002; 58: 135-141.

44. Buck LB. Olfactory receptors and odor coding in mammals. Nutr Rev 2004; 62 (11 Pt 2): 184-8; discussion 224-241.

45. Furudono Y, Sone Y, Takizawa K, et al. Relationship between peripheral receptor code and perceived odor quality. Chem Senses 2009; 34: 151-158.

46. Keller A, Zhuang H, Chi Q, et al. Genetic variation in a human odorant receptor alters odour perception. Nature 2007; 449 (7161): 468-472.

47. Zhou W, Chen D. Sociochemosensory and emotional functions: behavioral evidence for shared mechanisms. Psychol Sci 2009; 20: 1118-1124.

48. Albrecht J, Wiesmann M. The human olfactory system: Anatomy and physiology. Nervenarzt 2006; 77: 931-939.

49. Gudziol H, Wajnert B, Forster G. How do pleasant and unpleasant odours change the breathing pattern? Laryngorhinootologie 2006; 85: 567-572.

50. Pierce JD, Jr., Doty RL, Amoore JE. Analysis of position of trial sequence and type of diluent on the detection threshold for phenyl ethyl alcohol using a single staircase method. Percept Mot Skills 1996; 82: 451-458.

51. Tsukatani T, Miwa T, Furukawa M, et al. Detection thresholds for phenyl ethyl alcohol using serial dilutions in different solvents. Chem Senses 2003; 28: 25-32.

Rebekka Zernecke
Department of Neuroradiology
Ludwig-Maximilians-University of Munich
Marchioninistrasse 15
D-81377 Munich
Germany

Tel: +49-89-7095 5516

Fax: +49-89-7095 8719

E-mail: Rebekka.Zernecke@med.uni-muenchen.de 